Research Article

\title{
Aerodynamic and Vibration Characteristics of the Micro-Octocopter at Low Reynolds Number
}

\author{
Xiaohua Zou (iD, Mingsheng Ling, and Wenzheng Zhai \\ Changzhou College of Information Technology, Changzhou 213164, Jiangsu, China \\ Correspondence should be addressed to Xiaohua Zou; zouxiaohua@czcit.edu.cn
}

Received 7 May 2021; Revised 28 May 2021; Accepted 11 June 2021; Published 24 June 2021

Academic Editor: Sang-Bing Tsai

Copyright (C) 2021 Xiaohua Zou et al. This is an open access article distributed under the Creative Commons Attribution License, which permits unrestricted use, distribution, and reproduction in any medium, provided the original work is properly cited.

\begin{abstract}
With the development of flight technology, the need for stable aerodynamic and vibration performance of the aircraft in the civil and military fields has gradually increased. In this case, the requirements for aerodynamic and vibration characteristics of the aircraft have also been strengthened. The existing four-rotor aircraft carries limited airborne equipment and payload, while the current eight-rotor aircraft adopts a plane layout. The size of the propeller is generally fixed, including the load capacity. The upper and lower tower layout analyzed in this paper can effectively solve the problems of insufficient four-axis load and unstable aerodynamic and vibration performance of the existing eight-axis aircraft. This paper takes the miniature octorotor as the research object and studies the aerodynamic characteristics of the miniature octorotor at different low Reynolds numbers, different air pressures and thicknesses, and the lift coefficient and lift-to-drag ratio, as well as the vibration under different elastic moduli and air pressure characteristics. The research algorithm adopted in this paper is the numerical method of fluid-solid cohesion and the control equation of flow field analysis. The research results show that, with the increase in the Reynolds number within a certain range, the aerodynamic characteristics of the miniature octorotor gradually become better. When the elastic modulus is $2.5 \mathrm{E}$, the aircraft's specific performance is that the lift increases, the critical angle of attack increases, the drag decreases, the lift-to-drag ratio increases significantly, and the angle of attack decreases. However, the transition position of the flow around the airfoil surface is getting closer to the leading edge, and its state is more likely to transition from laminar flow to turbulent flow. When the unidirectional carbon fiber-reinforced thickness is $0.2 \mathrm{~mm}$ and the thin arc-shaped airfoil with the convex structure has a uniform thickness of $2.5 \%$ and a uniform curvature of $4.5 \%$, the aerodynamic and vibration characteristics of the octorotor aircraft are most beneficial to flight.
\end{abstract}

\section{Introduction}

The miniature octocopter has the functions of vertical takeoff and landing, hovering in the air and low-speed flight. Octorcopter aircraft is especially suitable for small environment to complete the specified work, its efficiency characteristics mainly depends on the wing, and the performance of the wing mainly depends on the shape of the wing, and its propeller Reynolds number belongs to the low Reynolds number range. Therefore, the development of high-efficiency rotors is of great significance for improving the endurance of the aircraft, the difficulty of control and the size of the load. For a miniature octocopter, it has a small rotor and a limited range, usually flying at a Reynolds number of 20,000 to 200,000. In the case of a small Reynolds number, the aerodynamic characteristics of the wing are significantly different from that of the wing when the Reynolds number is large, and the performance will also undergo complex changes.

Due to its own advantages, UAS plays an important role in high-tech local wars and performs very well in reconnaissance, environmental investigation, disaster prevention, and rescue missions in high-risk environments. Many scholars at home and abroad have also conducted in-depth studies on UAS. The performance is outstanding. Huang et al. used a low-speed wind tunnel to study advection and thin airfoils with Reynolds numbers ranging from 40,000 to 400,000 and pointed out that only by reducing airflow separation and reducing gas generation, airfoils can have better aerodynamics academic characteristics [1]. On the 
basis of Huang et al.'s research, Yuan et al. compared the aerodynamic characteristics of MDH5006 simple thin straight airfoil and thin round airfoil through manual experiments. The experiment showed that there are large differences in the magnitude of buoyancy [2]. Rahman et al. conducted tunnel wind experiments on Ep61 and Pf48 thin straight airfoils and thin arc airfoils. The research results show that the round thin arc airfoils have higher lifting force and lift-to-drag ratio, which is suitable for microaircraft [3]. Although the arc-shaped thin airfoil has better aerodynamic characteristics with small Reynolds number, the absolute thickness of the shape of the wing is not large because of the small size of the microrotor, so the structural stability and rigidity of the rotor are small. Chen et al. proposed that when the thin arc airfoil is applied to a microaircraft, it is not only easily damaged but also has poor aerodynamic stability [4].

The so-called Reynolds number is the size marked by the length of the diameter of the aircraft wing, and the general size is about 10,000 to 100,000 . Compared with the data flow of large Reynolds number, in the environment of small Reynolds number, the adsorption and nonsteady characteristics of the airflow field change significantly, resulting in the flow pattern around the wing generally showing the airflow pattern and resisting the back pressure gradient. There is a back pressure gradient in the airflow, the airflow around the wing is easy to block and transition, and then enter the jet stream state, which will have a significant impact on the aerodynamic characteristics of the wing. This requires studying the aerodynamic characteristics of small UAVs with low Reynolds numbers. Although increasing the thickness of the airfoil can improve the rigidity of the rotor structure, Josef et al. proposed a small Reynolds number airfoil with a downward concave structure [5]. Gawron et al. uses a numerical statistical method based on the three-dimensional fixed and non-decompressible orifice calculation formula to calculate the aerodynamic performance of the airfoil with a Reynolds number of 40,000 to 100,000 and compare it with the traditional arc thin airfoil [6]. Bari et al. studied the compressive index distribution area and airflow movement range of the airfoil at 60,000 hours. Finally, the airfoil was used to make a carbon fiber rotor with a diameter of $20 \mathrm{~cm}$ and a weight of 16 grams [7]. The hovering state was completed and the force was applied, and the architecture stability experiment was conducted. Ding et al. proposed the theoretical basis, experimental research and theoretical design of the low Reynolds number wing. In terms of experimental research, the low Reynolds number was studied through wind tunnel experiments, the flow reattachment was counted, and the pressure on the rotor surface of the aircraft was tested [8]. In terms of simulation analysis, Inui et al. used functional statistical methods to study the temperature-dependent characteristics of isolated bubbles and their effects on flight wing signs [9]. Colombo et al. used a digital model to study the sliding characteristics of equivalent flight wing signs in the case of a small Reynolds number and used an unsteady compressible viscous flow calculation model to numerically simulate the uneven airfoil in the case of a small Reynolds number [10].
This paper studies the aerodynamic and vibration characteristics of the micro-octorotor under low Reynolds number, discusses and analyzes the aircraft state under different elastic modulus and airflow environment, and studies the lift coefficient and drag coefficient of the microoctorotor with the upper and lower staggered towers. Liftto-drag ratio and the elastic modulus of the flight wing itself and the flight performance under different conditions. In addition, this paper studies the design of the eight-rotor flight system. By comparing the airfoil data, combined with the special conditions of the research object, the parameters of the best flight performance are determined according to the current situation, and various aspects of the model construction are successfully discussed. The innovation of this paper is that a different environment is set up to conduct aerodynamic and vibration research and analysis on the low Reynolds number miniature octorotor. The miniature octorotor is a new type of aircraft in the current aviation industry. Performance research contributes to the development of my country's aviation technology.

\section{Calculation Method of Aerodynamics and Vibration of the Miniature Octorotor}

2.1. Numerical Method of Fluid-Solid Cohesion. The interaction between airfoil and airflow is a typical problem of flexible connection of solid structures [11]. The deformation field changes according to the structure of the interface, and the extremely high distribution and pressure of the deformation field change according to the flow of the airfoil. The split line of airflow parameter transmission destroys the interaction of solsrfv architecture, and the interface of three key factors soon appears [12]. Using CXF software to calculate the smooth average Reynolds equation and the $\mathrm{S}$-shed jet flow calculation method, using the SST model as a prototype because the STK model has a better ability to capture the turbulent passage of airflow; the introduction of a new intermittent transition model can be expressed by the following equation:

$$
\begin{aligned}
\frac{\partial}{\partial t}\left(\rho_{\gamma}\right)+\frac{\partial}{\partial x_{j}}\left(\rho U_{j \gamma}\right)= & P_{\gamma 1}-E_{\gamma 1}-E_{\gamma 2} \\
& +\frac{\partial}{\partial_{x j}}\left[\left(\mu+\frac{\mu_{t}}{\sigma_{\gamma}}\right) \frac{\partial_{\gamma}}{\partial_{x j}}\right] .
\end{aligned}
$$

The reason for the intermittent period characterized by the equal space of the elements $\gamma$ is that the airflow cannot be stagnant and cannot remain stationary with the state unchanged, while $P \gamma$ and $E \gamma$ pass through the highly balanced space of the format, according to the sequence after a certain time discrete into the form of equations $[13,14]$. Ans is used to analyze the structure of the large slate domain architecture and perform solid analysis, so that the components that can drive the membrane are so soft, some of which are SH190 components, and are limited by capacity. The dispersion control equation is 


$$
M u^{\prime \prime}+C u^{\prime}+K u=F(t) .
$$

Among this parameter, $M, C$, and $K$ are mass linearity tables, damping linearity table and stiffness linearity table, respectively; $F(t)$ is the transient cohesion of the airflow acting on the architecture. The Hb-Jm multielectromagnetic field cohesion calculator MXF (multifield solver) is used to realize the airflow data transfer between the calculator and the solid calculator. In each time state of the state change process, it is applied to the flow field and the architecture respectively, and the interface data are calculated through multiple recursions until the recursive convergence condition is satisfied. The visual simulation calculation method is used in the airfoil adjustment process, which requires visual modeling of the wing fuselage structure and deformation parameters, and the use of function analysis tools is needed to adjust the parameters $[15,16]$. Among them, the $\mathrm{HiHe}$ function is used the most in tuning design. The HiHe-type function is based on the original airfoil, which can digitally characterize all possible wing postures in a given area. It is an intuitive and simple wing posture digitization method. Its general formula is

$$
\begin{aligned}
& Y_{1}(x)=Y_{10}(x)+\sum_{i=1}^{n} d_{1 i} \times f_{i}(x), \\
& Y_{2}(x)=Y_{20}(x)+\sum_{i=1}^{n} d_{2 i} \times f_{i}(x) .
\end{aligned}
$$

In the above formula, $Y_{1}$ and $Y_{2}$ are the upper and lower posture parameters of the wing posture; $Y_{10}$ and $Y_{20}$ are a function of the upper and lower surfaces of the reference wing body shape; $d_{i 1}$ and $d_{i 2}$ are the design variable of the upper and lower surfaces of the wing posture; $n$ is the number of design variables, and $f(x)$ is the control function corresponding to the design parameter.

The original HiHe function does not have a control function for the trailing edge of the wing posture, which makes the digital space for the trailing edge of the wing posture unable to expand, limiting the variety of wing posture adjustments. Therefore, the formula for adding a multitype functional function to the trailing edge of the wing posture is,

$$
\begin{aligned}
& f(x)= \begin{cases}x^{0.2}(1-x) e^{-20 x} & (i=1), \\
\sin ^{p}\left(\pi x^{e(q)}\right) & (1<i<n), \\
a x(1-x) e^{-\beta(1-x)} & (i=n),\end{cases} \\
& e(q)=\frac{\ln (0.5)}{\ln (q)}
\end{aligned}
$$

In the above formula, $P$ is the perturbation range parameter of the control function; $q$ is a one-dimensional array of length $n-2$, which controls the abscissa of the position corresponding to the peak of the $n-1$ contour function. The aerodynamic performance of the lower airfoil has a greater impact, so the design variable point is encrypted at the leading edge of the airfoil [17]. Take $q=\left[\begin{array}{llll}0.25 & 0.35 & 0.5 & 0.75\end{array}\right]$ and the change of the slope of the coefficient control function, the decay speed of the coefficient control function and other parameters depend on experience. Airfoil optimization mathematical model's airfoil aerodynamic performance determines the flight speed and energy efficiency of the aircraft, and the energy that a microaircraft can carry is limited, so energy efficiency is particularly important. For the rotor airfoil, the lift-to-drag ratio is the most important performance index, which has a great impact on improving the efficiency of the rotor [18]. Therefore, taking the lift-to-drag ratio of the rotor airfoil as the objective function, a generalized rotor airfoil objective function model is established:

$$
\text { objective: } \max \left(\frac{C_{E}}{C_{D}}\right) \text {. }
$$

Airfoil optimization methods can be divided into singleobjective optimization and multiobjective optimization. Single-objective optimization refers to the evaluation of only one target, only need to meet the specific function conditions, to obtain the maximum value. Multiobjective optimization refers to the existence of multiple evaluation functions, and the solution using different evaluation functions is different. That is to say: in the multiobjective optimization problem, there are multiple maximizing or minimizing objective functions at the same time, and these objective functions are not independent of each other, nor harmonious with each other. There will be more or less conflicts among them, so that all objective functions cannot be satisfied at the same time. The wing parameters need to be tested at different Reynolds conditions, and it needs to be compared to maintain better airflow efficiency at large elevation angles. In this case, multiobjective optimization is more appropriate. Therefore, the multiobjective optimization idea of the rotor airfoil under low Reynolds number can be expressed as the weight coefficient of the airfoil under different conditions. It is a vector corresponding to different design variables. The airfoil optimization process is based on the multiobjective optimization process of the rotor airfoil based on the improved genetic algorithm. The rotor algorithm is used to randomly generate the initial rotor, and the first generation airfoil set is generated within the design interval, and output to the wing the $X$-type foil aerodynamic solver calculates the performance of the airfoil set and then inputs the required data into the main program to calculate the fitness [19]. The accuracy and reliability of the $X$-type foil have been applied and verified. In the initial stage of optimization, in order to ensure the diversity of the rotors, a larger number of rotors and the probability of cross-mutation are required. In the later stage of optimization, the individual difference of the airfoil becomes smaller, and the operator and fitness function must be readjusted to ensure the continuous development of the rotor [20].

2.2. Flow Field Analysis Control Equation. The microrotor aircraft works at low Reynolds number, and the air viscosity has a great influence on it. Therefore, the influence of air viscosity must be considered when analyzing the 
aerodynamic characteristics of miniature quadrotor aircraft. Naiver Stokes equation is used as the control equation of the flow field because of its stable and incompressible characteristics.

The mass equation is

$$
\frac{\partial u}{\partial x}+\frac{\partial v}{\partial y}+\frac{\partial w}{\partial z}=0
$$

The momentum equations in the $X$ - and $Y$-direction are as follows:

$$
\begin{aligned}
& \rho\left(u \frac{\partial u}{\partial x}+v \frac{\partial u}{\partial y}\right)=\mu\left(\frac{\partial^{2} u}{\partial x^{2}}+\frac{\partial^{2} u}{\partial y^{2}}\right)-\frac{\partial p}{\partial x} \\
& \rho\left(u \frac{\partial v}{\partial x}+v \frac{\partial v}{\partial y}\right)=\mu\left(\frac{\partial^{2} v}{\partial x^{2}}+\frac{\partial^{2} v}{\partial y^{2}}\right)-\frac{\partial p}{\partial y}
\end{aligned}
$$

The energy equation is

$$
\frac{\partial E}{\partial x}+\frac{\partial E}{\partial y}=Q+W
$$

where $P$ is the fluid density, $u$ and $v$ are the components of the fluid velocity in $X$ - and $Y$-directions, respectively, $E$ is the total energy of the system, $p$ is the heat input into the system, and $\mu$ is the viscosity coefficient of airflow.

2.3. Shape and Structure of the Octorotor Aircraft. Traditional octorotor aircraft mainly use carbon fiber tubes as supports and use connecting plates to vertically fix the carbon fiber tubes and the flight controller, receiver, ESC, and battery of the octorotor aircraft. This method is convenient to obtain materials for the manufacture of eight-ring aromatic compounds and is compatible, but it is not windresistant and adaptable [21]. In order to improve the wind resistance and durability of the octagonal propeller aircraft, the manufactured octagonal propeller aircraft adopts composite film forming technology to produce a lightweight, strong wind resistance octagonal propeller aircraft structure. The integrated structure can effectively reduce the impact of natural factors on the aircraft [22]. For example, 8-bit mechanical and electronic equipment is not easily damaged in rainy weather and is itself waterproof. According to the bending, shearing, and twisting characteristics of the girders of the octagonal rotor, the parts are strengthened with greater force to ensure the safety of the fuselage. Since the inertia moment of the octagonal motor around the three axes, the motor inertia moment and the distance between the center of gravity of the motor, and the center of gravity of the aircraft are the main factors affecting the angular speed of the octagonal motor, the octagonal motor inertia moment controls the aircraft and reduces interference [23]. The moment of inertia of the aircraft around the three axes, the moment of inertia of the motor and the distance between the center of gravity of the motor and the center of gravity of the aircraft should be as small as possible. According to the overall characteristics of the octorotor aircraft, composite materials can be used for lamination, aramid paper honeycomb composite materials, glass and carbon fiber, and other materials can be used for lamination, and heating and vacuuming processes can be used for lamination. The fuselage can be integrated [24]. The molding uses a composite material connection process between the upper and lower bodies to produce an integrated 8-rotating body with high structural strength and lightweight. Under normal conditions, the efficiency number of a high-efficiency engine is about 20 grams per watt, and the rated output is 250 watts [25]. The maximum pulling force is $7.2 \mathrm{~kg}$. An eight-rotor aircraft has a mass of approximately $8 \mathrm{~kg}$ and a maximum flight load of only $16.4 \mathrm{~kg}$. The aircraft power unit is completely provided by the aircraft wing, and 8 identical subflight systems are arranged symmetrically and evenly to meet the load requirements of the aircraft. The high-speed plane flying engine is selected as the power source, and the propeller model is selected to obtain the maximum efficiency according to the current and pulling force of the electric motor [26]. The propeller model is APC15*4, it uses the selected motor to measure the relationship between motor output, lift, and efficiency at different speeds, different propellers and different motor outputs, so as to produce the highest efficiency. This choice can effectively improve the flight efficiency and durability of octets [27, 28].

\section{Design of Aerodynamic and Vibration Detection for the Microaircraft}

3.1. Research Objects. This experiment takes a miniature octorotor aircraft as the research object, adopts a staggered tower layout of upper and lower layers, and further researches on the basis of the existing four-axis multirotor aircraft with low carrying and external hanging capacity, adjustable thrust-to-weight ratio, and higher wind resistance. The technical solution adopted in the practical model includes 8 rotors including basic components, mechanical assembly, motor assembly, and running gears. The basic components are equipped with organic arm components and robotic arm components. The features of the practical model include that the upper cover plate of the machine base is round, the four convex rectangular pressing plates are evenly distributed in the circumferential direction, the fixed plate of the machine base is round, and the 8 rotors protrude equally in the circumferential direction.

\subsection{Design of Detection Steps}

3.2.1. Aerodynamic Testing Design of the Aircraft. The micro-air vehicle uses pneumatic control signals, and all differential pressure sensor signals are output from the filter. The output voltage signal is usually $\mathrm{mV}$ level of the piezoresistive sensor, and the output instrumentation amplifier amplifies the signal sensor. The maximum deviation of railto-rail output voltage is only $17 \mu \mathrm{V}$, and the noise reaches the peak value, that is, the peak value, and its frequency response range is $15 \mathrm{~Hz} \sim 0.017 \mu \mathrm{V}$, which is greater than $2.2 \mathrm{k} \Omega$. At the same time, if the symbols of the filter are gathered in the space of the SM2580 to perform the airflow area of the differential amplifier, the input radio frequency on the basis 
of the filter will be added to the airflow area of the amplifier if necessary. Similarly, if the implementation is not good, then any difference in the common mode of the converted standard input. There are at least 10 reference objects, so the micro-octocopter can suppress the mismatch level of the differential through the filter. The output signal of the instrument amplifier should be filtered to adjust the signal's instrument amplification to zero and use the MA295 chip in the filter. The universal 8 -order filter chip can reduce the error of pneumatic detection, and the operation is extremely simple. The cut-off frequency and clock frequency method can arbitrarily obtain the filter controlled by the same method clock, and the ratio of the cut-off frequency is $25: 1$.

3.2.2. Aircraft Vibration Detection Design. The experience of the airfoil controller using the aforementioned method of the controller, including the allocation of airfoil analysis algorithm resources and the pressure of the resultant force of the designed analyzer depends on the way of operation. The first two methods themselves have been widely used in practice. The structure of the thin elliptical arc-shaped wing surface at the upper edge of the lower edge of the triangular curved arc is curved on the back. The arc maintains the traditional wing structure, and convex surfaces are added to the upper wing to provide room for stiffening. It contains 8 parts of the airfoil, the first of which passes through the mouth line of the upper row of the arcuate upper bow. What follows is a curved concave arch shape that is connected to the top, and the result is to close the lower curved mouth to form an airfoil. To facilitate the calculation, the wings of the front end and back end are circular arc form, comparison is a small arc airfoil aerodynamic effect of the different measurement index, coefficient of airfoil increases coefficient increases with the decrease of the thickness and the increase of the thickness of the wing increases with the increase of vibration frequency, on a flat surface, the surface has the opposite concave near trailing edge, trailing edge thin, and bent down. When the airflow bypasses the leading edge of the ordinary airfoil, the velocity increases more and the velocity continues to increase on the upper surface of the airfoil. The thicker the airfoil, the more the upper surface bulges up, and the more the speed increases. At high enough speeds, the local flow rate on the upper surface of the airfoil reaches the speed of sound. Therefore, the thickness of the airfoil is more than 2 times the camber, and the aerodynamic performance is better. But at the same time, the strength of the airfoil structure has not decreased, and the difficulty and rigidity of processing it have not increased. The rotor checked the need for processing the fiber itself, and analyzed that a layer of fiber film and the internal coating itself were forced to maintain two unidirectional fiber ribs with a thickness of $0.3 \mathrm{~mm}$. In addition, the high performance of the safely placed aerodynamic airfoil has a thin and uniform arc structure thickness, and the convexity of the airfoil is designed to be uniform and elastic, varying within the elastic limit of $2.5 \%$ to $4.5 \%$.

\section{Aerodynamic and Vibration Characteristics of the Aircraft}

4.1. Aerodynamic Characteristics of Different Reynolds Numbers. First, the aerodynamic characteristics of the microairfoil under different air pressure models are calculated and analyzed. Figure 1 shows the relation between the lift-to-drag coefficient of the flexible wing and the angle of attack when the Reynolds number is 12,500 and the curing modulus is $2.25 \mathrm{MPa}$ and $1.5 \mathrm{MPa}$, and it is compared with the rigid wing. Before reducing the air pressure, the lift coefficient will increase linearly, and then decrease the air pressure greater than $0.8 \mathrm{MPa}$. When the lift coefficient of the rotor slows down, and the coefficient reaches the extreme value of the angle of attack of the wing, the lift coefficient increases by 0.8 .

As shown in Table 1, for such a wing with a modulus of elasticity of $2.5 \mathrm{MPa}$, the aerodynamic coefficient is not much different from that of a rigid wing at this time. The impulse angle of the airflow is 30 , and the impulse is greater than 0.8 . Because they destroy the growth rate, the angle of attack begins to increase. Compared with the rigid structure, when the deceleration rate of the rigid wing is reduced and the stall angle and lift are delayed, the $1.2 \mathrm{MPa}$ peak will increase by $4.5 \%$. Compared with the rigid wing, it has a maximum increase of $27 \%$ at a proper angle of attack $(\alpha=1.5)$ and has a drag coefficient to a certain extent. When the elastic mode of the wing with a coefficient of $2.5 \mathrm{MPa}$ does not occupy the highest price of the rise time, most of its corners will do its expansion, thereby further increasing the aerodynamic coefficient of the rhombic airfoil with vibration stability. The elastic modulus increases, and the surface characteristics of the flow airfoil in this area are still controlled by the steady flow.

After testing by the numerical solution method, the NE algorithm is used to determine the Reynolds of the wing of the micro-multirotor as $1.0 \times 10^{5}, 5 \times 10^{5}, 1.0 \times 10^{6}$, and the aerodynamic characteristics of the approach angle are calculated numerically, and the picking angle is within a certain range conduct statistical analysis. As shown in Figure 2, the relationship diagram of the blade lift index, inertial gravity, and lift-to-gravity ratio is obtained by numerical calculation. As can be seen from the figure, the smaller the incident angle is, the smaller the uplifting force index and boundary incident angle are. In other words, with the increase of the degree of AOA, regardless of positive or negative, the value of $\mathrm{Cd}$ will also increase. When $\mathrm{Cd}=1.36$ and $\mathrm{Aoa}=20$, the ratio of rising force index to inertial gravity is very large. When the pickup angle is 7 , the ratio of the rising force is most obvious. As the elevation angle increases, the wing transition area will move from the rear end to the front end along the rear edge. When the pickup angle reaches $17^{\circ}$, it reaches the vicinity of the front edge. When $\mathrm{Re}=5.0 \times 105$, the lift coefficient becomes linear at medium and small elevation angles and increases at small elevation angles. Both the maximum lift coefficient and the angle of incidence are very large, $\mathrm{Cd}=0.143$. When the drag coefficient is small and 


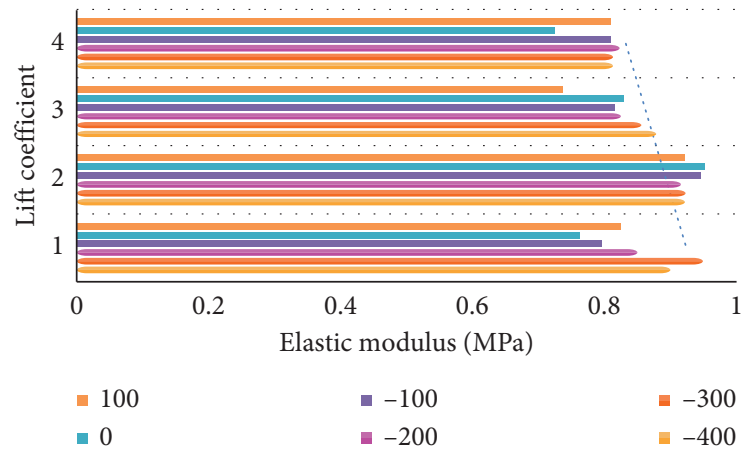

Figure 1: The lift coefficient values of the microairfoil under different air pressure models.

Table 1: Performance comparison between the optimized airfoil and initial airfoil under various Reynolds numbers.

\begin{tabular}{lcccccc}
\hline \multirow{2}{*}{ Rate } & \multicolumn{2}{c}{ NACA0012 } & \multicolumn{2}{c}{ New airfoil } & \multirow{2}{*}{ Rate change of C1 (\%) } & Rate change of C4 (\%) \\
& $\mathrm{Cl} 1$ & $\mathrm{Cd} 1$ & $\mathrm{Cl} 2$ & $\mathrm{Cd} 2$ & 21.06 & -24.87 \\
$\mathrm{Re}=100,000$ & 0.84082 & 0.0304 & 1.01788 & 0.02284 & 20.48 & -21.89 \\
$\operatorname{Re}=200,000$ & 0.85228 & 0.0222 & 1.02682 & 0.01734 & 20.59 & -15.77 \\
$\mathrm{Re}=300,000$ & 0.85994 & 0.01826 & 1.03698 & 0.01538 & \\
\hline
\end{tabular}

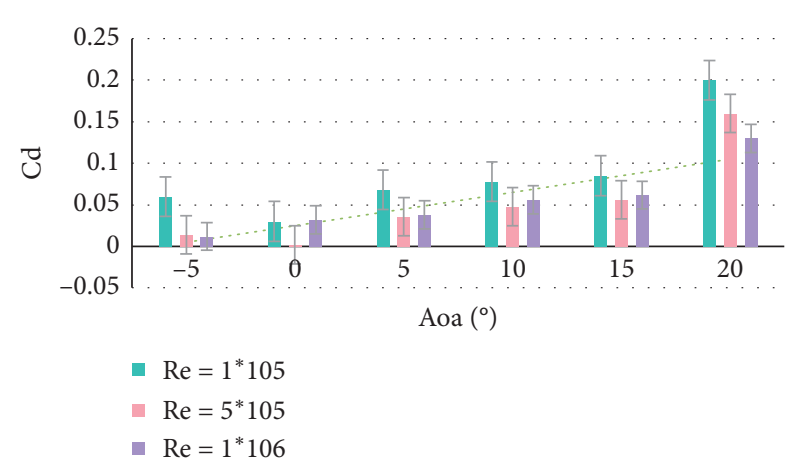

FIgure 2: Competitiveness curve.

the elevation angle is 4 , the lift ratio is the largest. As the elevation angle increases, the wing transition position will move along the rear surface from the trailing edge to the leading edge, so that the elevation angle Aoa is 12 . When you reach the forefront, if Re is $1.0 \times 10^{6}$, the lift coefficient, drag coefficient, lift reaction ratio, and the transition position change caused by the incident angle are basically the same when compared with $R e=5.0 \times 10^{5}$. Even if the incident angle is small, the value is basically the same. At high elevation angles, the drag coefficient is small, the lift coefficient is large, the critical elevation angle and the maximum lift/ reaction ratio are both large, $\mathrm{Cd}=0.152$. Generally, as the Reynolds number continues to increase, the aerodynamic characteristics of the winged clarke will gradually improve. The specific performance is that the lift increases, the critical elevation angle increases, the drag decreases, and the lift ratio increases significantly. Preferably, the elevation angle of the multirotor wing decreases. However, the transition position of the flow around the airfoil surface is getting closer and closer to the leading edge, and its state is more likely to transition from airflow to turbulent flow.
4.2. Vibration Characteristics of Angle Attack of the Flexible Wing. As shown in Figure 3, the lift coefficient increases with the increase of the wing camber within a certain range and decreases with the increase of the wing thickness, and the drag index increases with the increase of the thickness. As the curvature increases, it becomes smaller. Therefore, the smaller the wing thickness and the greater the camber angle, the better the aerodynamic performance of the octorotor. At this time, the structural stability and rigidity of the wing will be reduced to a certain extent, and the manufacturing difficulty will also increase. Since a layer of nanofibers needs to be embedded during production, this layer of material has a great influence on the fluidity of the wing, which is conducive to the improvement of efficiency. The relationship between the internal resistance of the motor and the no-load current is traded off. Select a high-efficiency motor directly by selecting a small internal resistance and a small no-load current. The motor efficiency is also closely related to the equivalent voltage and current. The control effect of the cascade fractional $\mathrm{Cd}$ is better than that of the cascade integer $\mathrm{Cd}$, the overshoot of both is close to zero, the cascade fractional $\mathrm{Cd}$ is adjusted to 0.18 , and the cascade integer $\mathrm{Cd}$ is more flexible. The system response simulation curve when the roll angle is limited to a certain range at random steps. The cascade fractional-order $\mathrm{Cd}$ response curve is always located between the input signal curve and the cascade integer-order $\mathrm{Cd}$ response curve, which indicates that the cascade fractional order $\mathrm{Cd}$ can track the change of the expected attitude and always maintain a stable and fast state, thus obtaining the close relationship between the motor and the current.

As shown in Figure 4, when the modulus of elasticity is 2.5 , whether it is a wing with a greater hardness or a wing with a less rigidity, the noncritical angle of attack flows on the suction surface, and the wing with a greater hardness is 


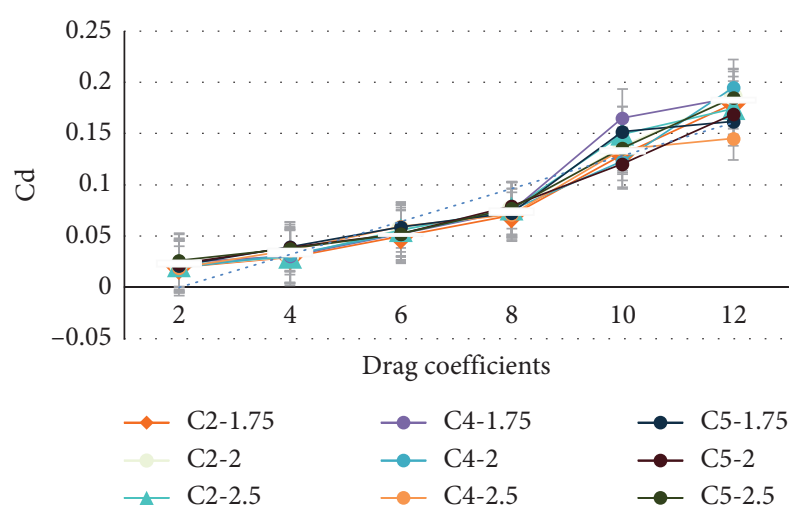

FIGURE 3: Lift-to-drag coefficient of the airfoil varies with the thickness.

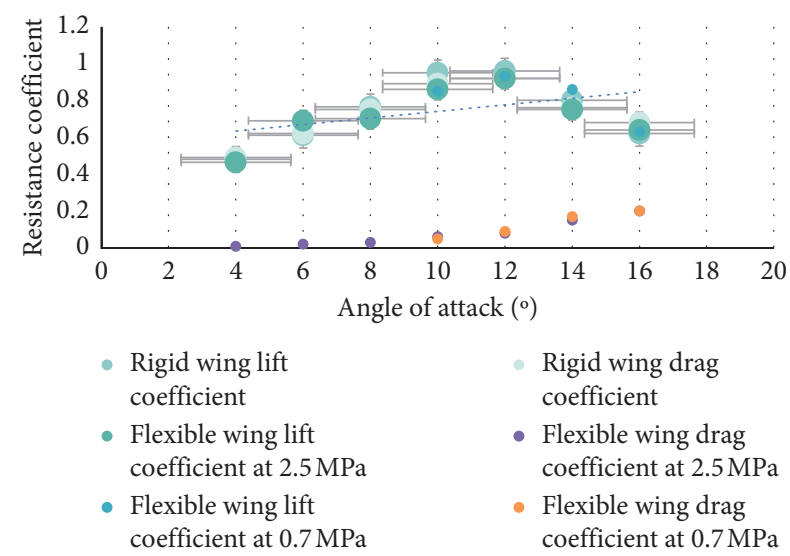

FIgURE 4: Lift and drag characteristics of flexible wings.

when the aerodynamic performance of the airfoil is not greatly affected, the leading edge separation flow is made to flow again, which greatly increases the rising force index. However, for a large critical angle of attack $\left(16^{\circ}\right)$, due to severe airflow separation, the influence of airfoil deformation is limited, the separated flow can no longer adhere, and the lift index increases insignificantly. When the modulus of elasticity is $0.7 \mathrm{E}$, the wing with greater rigidity will produce greater vibration, and the flow field on the airfoil surface has a strong unsteady flow phenomenon. When the modulus of elasticity is $0.7 \mathrm{E}$, the transient flow field of the airfoil surface at $13^{\circ}$ angle of attack is obtained. The large-amplitude vibration of the wing with a relatively hard middle makes the separated flow periodically adhere to the wing surface, and the vortex is rolled up on the wing surface. The vortex moves along the trailing edge of the wing and falls off, causing the lift index to fluctuate greatly. The second-level flexible airfoil nodes P1, P2, and P3 are all deformed to $2.5 \mathrm{E}$ at different angles of attack. The deformation of the wing with greater leading edge hardness is not obvious, and the deformation of the trailing edge is the most obvious. Before reaching the critical value, the time-average deformation of the two airfoil surfaces with less rigidity at the leading edge increases as the angle of attack increases, and the time-average deformation of the airfoil segment with the less rigid trailing edge decreases as the angle of attack increases. After the stall, as the angle of attack increases, the deformation of the two wing surfaces with less hardness at the leading edge gradually decreases, and the deformation of the wing with the less hardness at the trailing edge increases rapidly.

\section{Conclusions}

Microaircraft is a new type of aircraft that has emerged in recent years. Due to its low price and simple operation, it is widely used in military and civilian fields. At present, the optimization and debugging of microaircraft is in the climax of the world, and the related aerodynamic research is one of the frontiers of the research of micro-multirotor. MAV has small size, low operating speed, and small Reynolds number. Its entire flight loop is usually between tens of thousands to hundreds of thousands, and the Reynolds number is very low. If its Reynolds number is further reduced, its Reynolds number will be even lower, which is not available in previous conventional aircrafts.

The eight-rotor aircraft has the characteristics of large load demand, variable rotor size, and variable quantity. Currently, there are various rotor sizes available on the market, and there are many types of motors, and various rotor-motor matching schemes are available. How to quickly determine the power unit is one of the challenges in the development of micro-octorotors. When designing UAVs, the $4 / 6 / 8$ rotors are usually selected first. In order to ensure the application of the selected design in actual engineering, it is not only necessary to improve efficiency but also reduce the cost of components. Generally speaking, microaircraft can be divided into the following categories: fixed-wing model, flap model, rotor model, or a combination of the above three models. For flapping-wing aircraft, especially for eight-rotor aircraft, it is a more effective propulsion method than traditional multirotor aircraft. For the study of flap aerodynamic characteristics, a lot of research has been done in theoretical experiments and numerical simulations, but many studies are limited to the case of twodimensional airfoils. This article analyzes the impact of key factors such as aspect ratio, reduced frequency, initial frequency, and angle of attack, and provides a certain theoretical basis for the design.

Due to its flexibility, low cost, and broad application prospects, the miniature eight rotor has attracted many researchers. However, aerodynamics under low Reynolds number has always been a difficult point in research. The fluid-solid cohesion analysis is performed on the miniature octorotor, the flow field in the suspension is calculated numerically, and the structure is analyzed statically and modally. The closer the rotor flow field is to the rotor surface, the greater the wind speed and the larger the outer circle of the rotor along the rotor center. The maximum pressure in the flow field occurs at the tip of the blade. The state of the flow field has nothing to do with the flight attitude and the speed of the aircraft, it is mainly related to the rotation speed of the rotor. The maximum stress on the rotor surface occurs at the maximum thickness of the blade section. 
In addition, this article also has some shortcomings. Due to the limited cost and funds, this experiment only carried out simulation and did not conduct field research. There may also be some errors. It is hoped that through future research on miniature octorotors, we can gain further understanding.

\section{Data Availability}

The data that support the findings of this study are available from the corresponding author upon reasonable request.

\section{Conflicts of Interest}

The authors declare that they have no conflicts of interest.

\section{Acknowledgments}

This paper is the research results of the Provincial Engineering Research Center of Jiangsu (the Innovation and Application of Jiangsu Small Business Industrial Internet Engineering Research Center) and the Excellent Science and Technology Innovation Team of Jiangsu Universities and Colleges (the Application of Industrial Networks and Big Data). This research work was supported by Natural Science Research Project for Higher Education of Jiangsu (18KJB480001), Jiangsu Cyan Engineering in Young Academic Leaders 2019, Natural Science Research Project of CCIT (CXZK201805Q), Open Lab of Edge of Computing for Smart Manufacturing of CCIT (KYPT201802z), Changzhou Key Laboratory of High Technology (CM20183004), TopNotch Academic Programs Project of Jiangsu Higher Education Institutions (TAPP) (PPZY2015A090), and Laboratory of Application and Research of Artificial Intelligence Technology (PYPT201805Z).

\section{References}

[1] X. Huang, J. Sun, H. Hua et al., "Modeling and optimization of octostrut vibration isolation platform by FRF-based substructuring method," Journal of Aerospace Engineering, vol. 28, no. 3, pp. 1-11, 2015.

[2] W. Yuan, B. Yang, Y. Yang, K. Ren, J. Xu, and Y. Liao, "Development and experimental study of the characteristics of a prototype miniature vapor compression refrigerator," Applied Energy, vol. 143, no. 1, pp. 47-57, 2015.

[3] M. M. Rahman, M. Saha, M. M. K. Bhuiya, A. Biswas, M. H. Alam, and C. M. Feroz, "Heat transfer characteristics of a parallel miniature heat pipe system," International Communications in Heat and Mass Transfer, vol. 79, no. 7, pp. 1-8, 2016.

[4] J. Chen, Z. Zheng, and X. Lin, "Simulation analysis of stator vibration characteristics of miniature standing wave ultrasonic motor," Yadian Yu Shengguang/Piezoelectrics and Acoustooptics, vol. 40, no. 6, pp. 951-954, 2018.

[5] V. Josef, B. Zbynek, and M. Vaclav, "P92 and 15CH2NMFA steels-a comparison of fatigue characteristics obtained on standard and miniature test specimens," Procedia Engineering, vol. 213, no. 9, pp. 824-829, 2018.

[6] B. Gawron, T. Biaecki, and K. Artur, "The effect of adding 2ethylhexanol to jet fuel on the performance and combustion characteristics of a miniature turbojet engine," Journal of KONES, vol. 25, no. 1, pp. 101-110, 2018.

[7] H. A. A. Bari, Z. Yousif, Z. B. Yaacob, and E. Oluwasogaakindoyo, "Effect of SDBS on the drag reduction characteristics of polyacrylamide in a rotating disk apparatus," International Journal of Basic and Applied Sciences, vol. 4, no. 3, pp. 326-329, 2015.

[8] W. Ding, K. Wang, Z. Mao et al., "Layout optimization of an inertial energy harvester for miniature underwater mooring platforms," Marine Structures, vol. 69, no. 2, pp. 2681-2687, 2020.

[9] M. Inui, R. Sasaki, and T. Hamasaki, "Statistical analysis of manufacturer difference for miniature triode tube characteristics based on physical model parameters," Electronics and Communications in Japan, vol. 103, no. 8, pp. 9-20, 2020.

[10] M. L. Colombo, A. Fernández, C. V. Cimino et al., "Miniature cheeses made with blends of chymosin and a vegetable rennet from flowers of Silybum marianum: enzymatic characterization of the flower-coagulant peptidase," Food Chemistry, vol. 266, no. 15, pp. 223-231, 2018.

[11] L. Zhao, M. Yu, J. Fu et al., "A miniature MRE isolator for lateral vibration suppression of bridge monitoring equipment: design and verification," Smart Material Structures, vol. 26, no. 4, pp. 47-62, 2017.

[12] S. P. Jones and J. D. Delaurier, "Aerodynamic estimation techniques for aerostats and airships," Journal of Aircraft, vol. 20, no. 2, pp. 120-126, 2015.

[13] J. Cho and J. Cho, "Quasi-steady aerodynamic analysis of propeller-wing interaction," International Journal for $\mathrm{Nu}$ merical Methods in Fluids, vol. 30, no. 8, pp. 1027-1042, 2015.

[14] H. Chen, D. Fan, J. Huang, W. Huang, G. Zhang, and L. Huang, "Finite element analysis model on ultrasonic phased array technique for material defect time of flight diffraction detection," Science of Advanced Materials, vol. 12, no. 5, pp. 665-675, 2020.

[15] M. Amitay, D. R. Smith, V. Kibens et al., "Aerodynamic flow control over an unconventional airfoil using synthetic jet actuators," Aiaa Journal, vol. 39, no. 3, pp. 361-370, 2015.

[16] R.-H. Zhang, Z.-C. He, H.-W. Wang, F. You, and K.-N. Li, "Study on self-tuning tyre friction control for developing main-servo loop integrated chassis control system," Ieee Access, vol. 5, pp. 6649-6660, 2017.

[17] G. Xu, X. Liang, S. Yao, D. Chen, and Z Li, "Multi-objective aerodynamic optimization of the streamlined shape of highspeed trains based on the kriging model," PLoS One, vol. 12, no. 1, Article ID e0170803, 2017.

[18] D. Hummel and P. S. Srinivasan, "Vortex breakdown effects on the low-speed aerodynamic characteristics of slender delta wings in symmetrical flow," The Journal of the Royal Aeronautical Society, vol. 71, no. 676, pp. 319-322, 2016.

[19] R. May, O. Reitan, K. Bevanger, S.-H. Lorentsen, and T. Nygård, "Mitigating wind-turbine induced avian mortality: sensory, aerodynamic and cognitive constraints and options," Renewable and Sustainable Energy Reviews, vol. 42, no. 7, pp. 170-181, 2015.

[20] M. Pingchang, L. Yan, and H. Li, “Aerodynamic design and optimization of high altitude environment simulation system based on CFD," AIP Conference Proceedings, vol. 1839, no. 1, pp. 1-7, 2017.

[21] C. Xinghua, Z. Laiping, and R. Ma, "Numerical investigation on aerodynamic performance of a bionic flapping wing," Applied Mathematics and Mechanics (English Edition), vol. 40, no. 11, pp. 97-118, 2019. 
[22] T. C. Corke and F. O. Thomas, "Dynamic stall in pitching airfoils: aerodynamic damping and compressibility effects," Annual Review of Fluid Mechanics, vol. 47, no. 1, pp. 479-505, 2015.

[23] D. J. Thompson, E. Latorre Iglesias, X. Liu, J. Zhu, and Z. Hu, "Recent developments in the prediction and control of aerodynamic noise from high-speed trains," International Journal of Rail Transportation, vol. 3, no. 3, pp. 119-150, 2015.

[24] S. D. Kim, "Aerodynamic design of a supersonic inlet with a parametric bump," Journal of Aircraft, vol. 46, no. 1, pp. 198-202, 2015.

[25] J. P. Loomis and R. F. Porter, "Examination of an aerodynamic coupling phenomenon," Journal of Aircraft, vol. 2, no. 6, pp. 553-556, 2015.

[26] R. J. Cattolica, R. J. Gallagher, J. B. Anderson et al., “Aerodynamic separation of gases by velocity slip in freejet expansions," AIAA Journal, vol. 17, no. 4, pp. 344-355, 2015.

[27] W. E. Hall and N. K. Gupta, "System identification for nonlinear aerodynamic flight regimes," Journal of Spacecraft \& Rockets, vol. 14, no. 2, pp. 73-80, 2015.

[28] W. Yong, "Study on aerodynamic characteristics of supersonic airfoil," Modern Mechanical Engineering, vol. 09, no. 1, pp. 13-19, 2019. 